\title{
Case Report: Effect of a Long-Term Corrective Ex- ercises With Using Brace on Cobb Angle and Pos- tural Control of a Girl With Adolescence Idiopathic Scoliosis
}

\author{
Mehdi Gheitasi $^{1}$ (D), Mohammad Bayattork ${ }^{2 *}$ (D), Seyed Mani Mahdavi ${ }^{3}$ (D) \\ 1. Department of Health and Sport Rehabilitation, Faculty of Sport Sciences and Health, Shahid Beheshti University, Tehra, Iran. \\ 2. Department of Physical Education and Sport Sciences, Faculty of Humanities, University of Hormozgan, Bandar Abbas, Iran. \\ 3. Department of Orthopedics, School of Medicine, Iran University of Medical Sciences, Tehran, Iran.
}

\begin{tabular}{|c|c|}
\hline $\begin{array}{l}\text { Use your device to scan } \\
\text { and read the article online }\end{array}$ & Coftat Gheitasi M. Bavattork M. Mahdavi SM Effect of a Lono-term Corrective Exercises With Using Brace on Cobb \\
\hline aritipa & $\begin{array}{l}\text { Angle and Postural Control of a Girl With Adolescence Idiopathic Scoliosis: A Case Report. Physical Treatments. 2019; 9(1):59- } \\
\text { 68. https://doi.org/10.32598/PTJ.9.1.59 }\end{array}$ \\
\hline altar. & doi https://doi.org/10.32598/PTJ.9.1.59 \\
\hline
\end{tabular}

\section{(i) (5)}

Article info:

Received: 16 May 2018

Accepted: 24 Oct 2018

Available Online: 01 Jan 2019

Keywords:

Exercise therapy,

Adolescence idiopathic scoliosis, Balance, Posture, Braces

\begin{abstract}
A B S T R A C T
Purpose: Scoliosis is a three-dimensional deformity of the spine in which the resulting imbalance accompanied by the changes in muscle and nervous components can harm the postural control. Various treatments have been suggested so far to correct scoliosis, in which using corrective exercises along with brace are essential to limit the growing curvature, improve spine stability and postural control, and reduce the degree of curvature.

Methods: Our case was a 14-year-old girl who had a 40-degree thoracic curve (T6 to T11) toward the right and a 50-degree lumbar curvature (T12 to L4) toward the left. To evaluate her scoliosis, we took side view radiography for 4 to 6 months. Also, we used balance master posturography to assess the patient's postural control before and after the intervention. The patient's treatment process included a long-term implementation of a corrective exercise protocol in 3 phases plus using the Milwaukee brace.

Results: The Cobb angle in the kyphosis curve showed a 15-degree improvement. Also, the Cobb angle showed a 32-degree correction in the thoracic curve and a 30-degree reduction in the lumbar curve. A significant improvement was observed in all of the tests of postural control, indicating improved balance and postural control of the subject during corrective exercises.

Conclusion: Simultaneous using the brace and long-term corrective exercises with emphasis on the improvement of postural control could be useful in improving the scoliosis curves in an adolescent girl with idiopathic scoliosis.
\end{abstract}

\footnotetext{
* Corresponding Author:

Mohammad Bayattork, MSc.

Address: Department of Physical Education and Sport Sciences, Faculty of Humanities, University of Hormozgan, Bandar Abbas, Iran.

Phone: +98 (21) 88464537

E-mail: mohammadbayat82@gmail.com
} 


\section{Highlights}

- A long-term corrective exercises program with using brace improves thoracic kyphosis curve in a 14-year-girl with adolescence idiopathic scoliosis.

- A long-term corrective exercises program with using brace improves postural control factors in a 14-year-girl with adolescence idiopathic scoliosis.

\section{Plain Language Summary}

The main goals of all therapeutic interventions are to restrict or stop the spine curvature increase in scoliosis and its consequences. This case study showed that simultaneous use of a brace and long-term corrective exercises with an emphasis on the development of postural control variables could be useful in correcting the scoliosis curves in an adolescent girl with idiopathic scoliosis.

\section{Introduction}

coliosis is a three-Dimensional (3D) spinal deformity that includes a lateral curvature in frontal plate, vertebral rotation in transverse plate, and possible increase or decrease of thoracic and lumbar curves in sagittal plane. As a result of this deformity, the chest geometry and the symmetry of the trunk changes $[1,2]$. Changes in appearance and, in particular, elevation of the ribs and the scapula are the most important causes of dissatisfaction among people with Scoliosis, especially adolescents [3]. The most common type of scoliosis (80-90\%) is idiopathic scoliosis, whose aetiology is unclear. One of its types is Adolescent Idiopathic Scoliosis (AIS) that occurs during adolescence, the rapid growth period [4]. Many theories have been presented for the aetiology of AIS, including neuromuscular, growth, and genetic theories [5].

All hypotheses related to AIS sum up as follows: "The normal spine in a growing person has a precise, precarious, delicate mechanical balance. Asymmetrical changes in primary structures, support structures, growth centers, the position of the spine, and related neural or muscular components can all result in the development of scoliosis" [1]. The lack of symmetry, along with changes in neuromuscular components, can change the position of the center of gravity and the distribution of weight on the lower limbs. Therefore, it can be claimed that patients with scoliosis have a lower balance and postural control than their peers [6]. Hence, there is a possibility of postural control disorder and, consequently, the likelihood of exacerbation of abnormalities or incidence of new abnormalities and their complications in people with scoliosis [7].
In general, some studies have reported that people with scoliosis have a weaker postural control than those with normal anatomy, especially when their visual and sensory-motor systems are irritated. Also, some studies indicate that the postural stability of people with scoliosis is less than those with normal spinal curves, and they have lower balance than healthy people. This is characterized by increased posture sway in them relative to normal people [8]. Although little studies have been carried out on this relation, the contradictions and controversies between the previous studies are remarkable.

The treatment of idiopathic scoliosis depends on the characteristics of deformity. Since the curve progression in idiopathic scoliosis develops in most adolescents, the main goals of all therapeutic interventions are to restrict or stop curve progression, to restore trunk balance, and to prevent its long-term complications [9-11]. Currently, the common therapeutic interventions for AIS include scoliosis-specific exercises, bracing, and surgery [12] Of these, scoliosis-specific exercises are more accepted and preferred by adolescents with scoliosis and their parents than other methods $[13,14]$. The scoliosis-specific exercises are usually used to treat scoliosis curves of less than $25^{\circ}$ or $30^{\circ}$; however, this approach can be combined with bracing technique to be used for curves with higher Cobb angles [15].

Bracing is the use of an external support for the trunk. Braces are often rigid and used to achieve maximum correction of scoliosis curve. The mechanical forces created by the brace are used to straighten the spine and prevent the pelvis and shoulder rotation in order to put the whole body in the desired alignment [1]. Previous studies have shown that the external and proprioceptive inputs resulted from bracing change the unnatural loads applied 
to the spine and ribs, reduce asymmetrical movements, and improve neuromuscular control. These all lead to the proper spinal growth, neuromotor reorganization, and changes in motor responses [16-18].

The use of brace is usually recommended for when the curve is progressive or its Cobb angle has reached $30^{\circ}$ $[15,19]$. However, if brace is used without corrective exercises, there is a possibility of trunk muscles weakness over time [1]. In some studies, increased Cobb angle has also been reported in subjects, using only brace treatment $[20,21]$. In this regard, the aim of this study was to investigate the simultaneous effect of a long-term corrective exercise program and the use of Milwaukee brace on Cobb angle and postural control of a teenage girl with idiopathic scoliosis.

\section{Materials and Methods}

The present study is a case study that lasted 22 months. It was started from February 10, 2016 when the patient with idiopathic scoliosis was referred to the clinic of corrective exercises at Red Crescent Rehabilitation Center in Tehran with her parents, while having a booklet containing training and corrective exercises related to scoliosis given by an orthopedic and spine surgeon as well as the X-rays of the lumbar spine (anteroposterior and lateral views). At the initial meeting, her comprehensive posture assessment was carried out by a corrective exercise specialist. It should be mentioned that, according to the specialist and under the supervision of an orthotics expert, the use of the Milwaukee brace had been recommended.

Considering the patient's history, in addition to 22-hour use of brace, a corrective exercise program was designed based on radiographic images and assessment of physical condition including the evaluation of trunk symmetry and deformities of trunk, pelvis, and shoulder areas as well as anteroposterior curvature of thoracic kyphosis and lumbar lordosis, and lower extremities, including leg posture and knee alignment. The patient performed corrective exercises (details are provided in the next sections) under full supervision until February 10, 2018. During 4 to 6 months, she was re-evaluated by a spine specialist; then, she was radiographed and, if needed, she received brace correction.

\section{Study sample}

The study sample was a 14-year-old female (at the time of entering the study), who had idiopathic scoliosis (sshaped or double curve) according to an orthopedic and spine surgeon's diagnosis. She had no history of chronic diseases or orthopedic damages to the spine and lower limb, and in her family, there was no history of spinal cord disease and, particularly, the history of scoliosis. She was an 8th grade student, who, according to Iran's Education System, spends about 7 hours a day (on average) at school sitting at her desk. She performs most of her daily activities normally with no complaint or pain. Her parents accidentally noticed the asymmetry in the posterior side of her trunk, and after referring to a doctor and taking X-rays on June 25, 2015, they realized that she had scoliosis. Based on the determination of Cobb angle using the X-rays of the entire spine in the anteroposterior view, she had a 35-degree thoracic curvature (T6 to T11) and a 34-degree lumbar curvature (T12 to L4). However, despite the presence of scoliosis and the recommendations of the physician, no proper follow-up was performed by the patient and her parents. Seven months later, the X-rays were taken at the same center with view similar to the previous X-rays. The scoliosis curves at this time (after about 7 months) had a significant progression, and the person returned to the spinal surgeon, where bracing and corrective exercises were prescribed.

\section{Assessment}

In this study, the Cobb angle was measured by processing the anteroposterior and lateral X-rays according to the physician's instructions. The observational posture of the subject was assessed to determine the symmetry and alignment of the shoulders and pelvis, thoracic and lumbar protrusions (humps), and the evaluation of postural control, using a posturography device (Balance Master, NeuroCom Inc, USA). This device is a gold standard for the diagnosis of balance problems with clear and unclear aetiology. With this device, the ability of patient in using visual, vestibular, and somatosensory information is evaluated. This machine, by manipulating the sensory inputs needed for postural control and maintaining balance in 6 different positions, assesses the efficiency of each of these sensory resources in receiving and transmitting sensory data to the central nervous system under perturbations with open and closed eyes. It includes a dynamic force plate capable of rotation used to quantify the vertical forces of the patient's foot in order to measure the movement of the center of mass and the postural control. There is also a moving plate around the device to measure the patient's ability to use visual information to maintain balance.

In the present study, 5 assessment protocols, using this device, were employed to check the postural con- 
trol of the patient, which include Sensory Organization Test (SOT), adaptability test, weight-bearing test during squat, One-Legged Stance Test (OLST), and motion control test. Posture control assessments were performed at the beginning and at the end of the intervention.

In the first stage of evaluation conducted by processing the first X-rays on February 01, 2016, it was revealed that the patient had a 40-degree thoracic curvature (T6 to T11) toward the right and a 50-degree lumbar curvature (T12 to L4) toward the left. An asymmetry in the pelvic area was also observed, where the right pelvis was $8 \mathrm{~mm}$ higher than the left pelvis. The left shoulder was slightly lower than the right shoulder; this asymmetry and non-alignment in the pelvic and shoulder areas were consistent with the patient's scoliosis curves pattern. The vertebral rotation to the left in the thoracic region, and to the right in lumbar region was clearly visible such that with the implementation of Adam's bending test, a rib hump was observed in the right thoracic curve and in the left thoracic-lumbar curve. A slight increase was also observed in the spinal curve $\left(45^{\circ}\right)$ from the side view. Also, according to the diagnosis of relevant specialist, the vertebral growth plates were not completed at this time (Risser sign=3). Additional tests for assessing the range of motion in the spine, shoulders, and pelvis showed a natural state. According to the recommendations of the physician, individual assessments were repeated every 4 to 6 months to receive appropriate feedback for brace modification.

\section{Intervention}

The treatment process began from February 18, 2016 with the prescription of brace and continued by referral to the clinic of corrective exercises at Red Crescent
Rehabilitation Center for performing training program. The used brace was a Milwaukee brace, whose condition was monitored during treatment under the supervision of a specialist and according to radiographic images, and if necessary, modifications were made. Generally, brace is required to be worn for a significant period (at least 20 hours) and the bracing treatment usually often lasts several years; that is, until the bone growth stops. This time is almost 16 years for girls and 18 years for boys [22]. According to the spine specialist, the use of brace in the first week was 8 hours a day, and from the second week every day, 2 hours was added to the time of use. At the end of the third week, it reached about 22 hours. The patient was allowed to open the brace 1 hour for bathing and 1 hour for performing corrective exercises.

The corrective exercises were designed in 3 phases; at the first phase, the subject became familiar with her abnormality and subsequent musculoskeletal changes [23]. The exercises in this phase mostly focused on increasing the subject's awareness of being in an appropriate and symmetrical physical condition. Exercise for increasing spine length and core stability was also carried out in this phase. Previous studies have already shown the need for exercises to increase the spin length and core stability in adolescents with idiopathic scoliosis [24, 25]. At the early stages, only isometric muscle contractions were taken in static positions along with maintaining the proper alignment of the trunk, and the patient tried to control the target muscles consciously, so that, at the end, it can improve the proprioceptive sense and normalize the alignment of head, shoulders, spine, and pelvis. The exercises in this phase included 4 stretching and trunk lengthening exercises [26] and 4 core stability exercises

Table 1. Strengthening/stretching exercises (the first phase of intervention)

\begin{tabular}{cc}
\hline Exercises & Details \\
\hline 1 & Stretching spine and trunk while sitting in a chair \\
2 & Rolling trunk (stretching trunk down and forward using an exercise roll). \\
3 & Symmetric stretching of the trunk and spine in standing position \\
4 & Isometric contraction of the abdominal muscles while lying down \\
6 & Isometric contraction of the abdominal muscles with opposite arm/leg raise \\
7 & Supine bridge (co-contradiction of the trunk core muscles) \\
\hline
\end{tabular}


Table 2. Auto-correction/3D exercises (the second phase of intervention)

\begin{tabular}{|c|c|}
\hline Exercises & Details \\
\hline 1 & Simple 3D exercise in a lying position \\
\hline 2 & Self-correction in sitting position (SEAS exercise 1) \\
\hline 3 & Self-correction in standing position (SEAS exercise 2) \\
\hline 4 & Self-correction while leaning forward (SEAS exercise 3) \\
\hline 5 & Self-correction while sitting on a chair with a pole planted on the ground (SEAS exercise 4) \\
\hline 6 & Stretching out of the training ladder and correcting the spinal alignment (Schroth exercise 1) \\
\hline 7 & Muscle cylinder in standing position (Schroth exercise 2) \\
\hline 8 & Hip strengthening exercise in the front of a training ladder (Schroth exercise 3) \\
\hline 9 & Bending the thigh in side-lying position (Schroth exercise 4) \\
\hline 10 & Pulling the shoulder back to inside+ muscle cylinder in side-lying position (Schroth exercise 5) \\
\hline
\end{tabular}

PHYSICAL TREA $\ M E N T S$

(Table 1). The subject performed them under supervision for 3 months at 3 sessions.

At the second phase, the patient performed auto-correction and 3D exercises consistent with Schroth exercises and Scientific Exercise Approach to Scoliosis (SEAS). Recently, there has been some positive evidences in support of SEAS. Schroth exercises also have been used in many studies. In both methods, one of the most important goals of the exercises is to train the subject to restore the body to a suitable condition $[27,28]$. At the beginning of this phase, the subject had isometric muscle contractions for auto-correction of the curves by returning the curved spin back to normal position and reducing the vertebral rotation without bearing weight. Over time and with the patient's more mastery on how to perform self-correction in different positions, the state of exercise was changed to sitting and standing. Also, $3 \mathrm{D}$ exercises were based on exercises designed in 3 movement directions [26]. The exercises in this phase included 10 self-correction and $3 \mathrm{D}$ exercises for 12 months (Table 2).
At the third phase and after mastering how to perform auto-correction and 3D exercises, the patient performed functional exercises; that is, she performed self-correction in different positions on balance boards, practice balls, or while walking. This phase was designed to engage ever more proprioceptive sense receptors in exercises, to transfer the posture's correctional states from static to dynamic, and finally to transfer the correct posture to the daily living activities. The exercises in this phase included 6 exercises lasting 6 months (Table $3)$. Although the number of exercises at this phase was lower compared to the other phases, considering the high number of repetitions, the duration of exercises, and the amount of exercises at this phase was more. All exercises were performed during the treatment period (22 months), at least 3 sessions per week under the supervision of a corrective exercise specialist and 2 sessions per week at home.

Table 3. Functional exercises combined with daily tasks

\begin{tabular}{cr}
\hline Exercises & Details \\
\hline 1 & Balance and sensory-motor exercises on balance board and unstable surfaces \\
2 & Positioning the spin in dynamic physical conditions such as wall push-up and single leg stance (SEAS exercise) \\
3 & 3D correction of spinal alignment in sitting position (on chair/ Swiss ball) \\
5 & 3D correction of spinal alignment in sitting position (on stable/unstable surfaces) \\
6 & Schroth walking \\
\hline
\end{tabular}




\section{Results}

\section{Cobb angles in sagittal and frontal plates}

Based on the processing of X-ray images (from February 01, 2016 to November 22, 2017), the Cobb angle of the thoracic kyphosis in the patient showed a decrease of $15^{\circ}$ at the end of the treatment period. Moreover, the Cobb angle of upper scoliosis reduced as $32^{\circ}$ and for lower scoliosis curve, it showed a 30-degree decrease. The results of Cobb angle measurement in sagittal and frontal plates during the treatment period are presented in Table 4. The X-rays of kyphosis and scoliosis curves of the patient before and after intervention are shown in Figure 1. The measurement of Cobb angles by the specialist and clinical therapist in pre- and post-test phases was conducted in a 4- to 6-month interval.
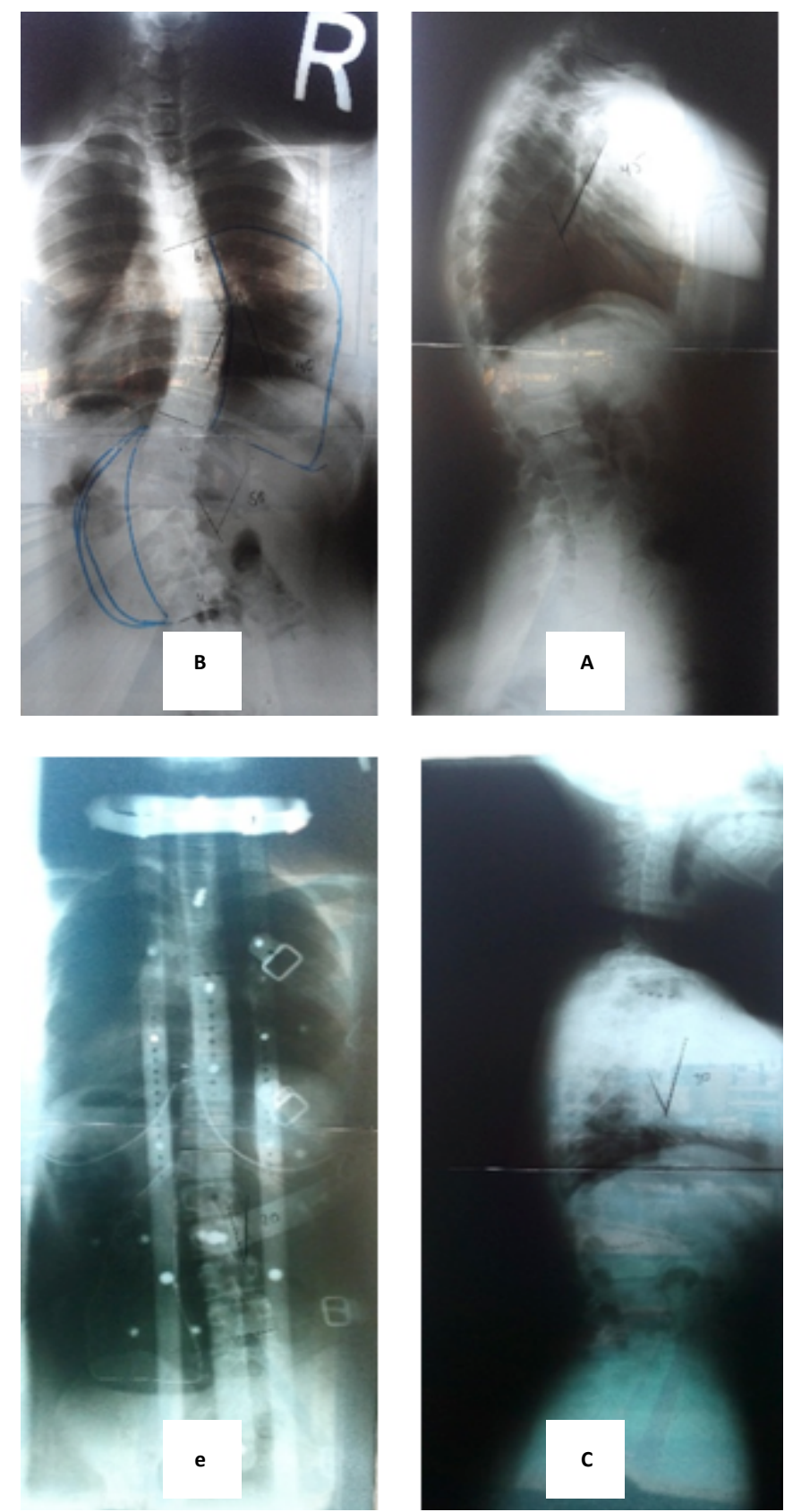

Figure 1. X-rays of Kyphosis and scoliosis curves before (a \& b) and after intervention (c \& d)

A. 45-degree thoracic kyphosis; B. 40-degree thoracic scoliosis and 50-degree lumbar scoliosis; C. 30-degree thoracic kyphosis; E. 10-degree thoracic scoliosis and 20-degree lumbar scoliosis 
Table 4. Changes in Cobb's angles in radiographic assessments

\begin{tabular}{|c|c|c|c|c|c|}
\hline \multirow{2}{*}{ Cobb Angle } & \multicolumn{5}{|c|}{ Radiographic Examination Period } \\
\hline & Feb 01, 2016 & Jul 13, 2016 & Nov 13, 2016 & Jul 24, 2017 & Nov 22, 2017 \\
\hline Cobb angle of thoracic kyphosis & $45^{\circ}$ & $45^{\circ}$ & $30^{\circ}$ & $30^{\circ}$ & $30^{\circ}$ \\
\hline Cobb angle of thoracic scoliosis & $42^{\circ}$ & $42^{\circ}$ & $35^{\circ}$ & $30^{\circ}$ & $10^{\circ}$ \\
\hline Cobb angle of lumbar scoliosis & $50^{\circ}$ & $45^{\circ}$ & $34^{\circ}$ & $36^{\circ}$ & $20^{\circ}$ \\
\hline
\end{tabular}

PHYSICAL TREA $\$ MENTS

\section{Static and dynamic balance and postural control}

The results of testing postural control and static and dynamic balance of the subject, using 5 methods measured by Balance Master System, are presented in Table 5. The SOT results showed a significant increase in the patient's balance scores, indicating improved balance and postural control after performing corrective exercises. Motion control test results showed a decrease in latency response to motor perturbations. The magnitude of motor response to perturbations under adaptability test was also reduced. These changes indicate an increase in the patient's ability to create a fast and proper motor response to external perturbations disturbing the balance. Furthermore, according to weight-bearing test results, no changes in the status of the patient have been made before and after intervention, but in both stages, her condition in bearing weight was normal. Under OLST, her sway velocity was significantly reduced, which can be indicative of her increased ability to maintain balance and control the posture when standing on one leg.

\section{Discussion}

The aim of this study was to evaluate the effectiveness of a long-term corrective exercises along with wearing Milwaukee brace on reducing the Cobb angle and improving the postural control of an adolescent girl with idiopathic scoliosis. The results of the study showed a significant improvement in Cobb angle in sagittal (ky-

Table 5. Balance and postural control test results

\begin{tabular}{|c|c|c|c|}
\hline Balance and Postural Control Tests & & At the Beginning & At the End \\
\hline \multirow{3}{*}{ SOT } & Total balance & 58 & 71 \\
\hline & Static balance & 90.2 & 93 \\
\hline & Dynamic balance & 40.3 & 59.4 \\
\hline Motion control test (Latency in ms) & & 150 & 140 \\
\hline Adaptability test (magnitude of motor response to perturbations) & & 50.2 & 48.8 \\
\hline \multirow{6}{*}{$\begin{array}{l}\text { Weight-bearing test during squat (percentage of weight-bearing on } \\
\text { both legs) }\end{array}$} & \multirow{2}{*}{$30^{\circ}$} & Right leg=45 & Right leg $=45$ \\
\hline & & Left leg=55 & Left leg $=55$ \\
\hline & \multirow{2}{*}{$60^{\circ}$} & Right leg=47 & Right leg=47 \\
\hline & & Left leg=53 & Left leg $=53$ \\
\hline & \multirow{2}{*}{$90^{\circ}$} & Right leg=46 & Right leg $=46$ \\
\hline & & Left leg=54 & Left leg $=54$ \\
\hline \multirow{5}{*}{ OLST (sway velocity in degrees/s) } & Left foot/eyes open & 1 & 0.7 \\
\hline & Left foot/eyes closed & 2.3 & 1.5 \\
\hline & & & \\
\hline & Right foot/eyes open & 0.8 & 1.2 \\
\hline & Right foot/eyes closed & 2.6 & 2.4 \\
\hline
\end{tabular}


phosis) and frontal (scoliosis) plates. The goal of AIS treatment is to prevent the development of curvature by up to $50^{\circ}$, where there is a risk of increase in adulthood. Therefore, if the curvature reaches $50^{\circ}$ during adolescence, surgery is generally recommended as a therapeutic method [29]. However, many affected adolescent and their parents tend to have therapies other than surgery. One of these methods is bracing, which is an accepted technique for preventing curvature progression [30]. However, the success rate of bracing has been reported $72 \%$ [13]; this means that many people with scoliosis, despite bracing and if they fail to slow or control the progression of curvature after the growth period and closure of spinal growth plates, have to undergo surgery. Meanwhile, scoliosis-specific exercises are well accepted by adolescents with idiopathic scoliosis and their parents [13]. Several randomized controlled trials have reported positive effects of corrective exercises on reducing curvature progression and improving trunk cosmetic appearance and quality of life $[14,31,32]$, although in these studies, subjects had different characteristics and were heterogeneous. For example, subjects were at different skeletal maturity stages, or some studies used non-standard methods with low reliability for measurement. Therefore, the effectiveness of these exercises remains unclear when the curvature progression is high [29].

Many similar studies have either focused on the effectiveness of exercises or have only examined the effects of using brace $[12,27]$. Only a few studies have examined the effectiveness of bracing combined with corrective exercises in long-term periods [14, 29]. Among these studies, only Kwan et al.'s study examined the effect of exercises during bracing [29]. Zheng et al. only compared the effectiveness of bracing and exercises in patients with AIS and finally reported significant improvement in each study group [33]. According to the results of the previous studies, it seems that the best nonsurgical treatment for AIS is the simultaneous use of exercises and bracing $[3,34]$.

Based on the results of the present study, during 22 months of performing corrective exercises under specialist's supervision and simultaneously wearing the Milwaukee brace, the degree of scoliosis curve in the patient significantly decreased. This result is consistent with the results of a study conducted by Negrini et al. [35]. In this study, the degree of scoliosis in the patient was reported $42^{\circ}$ for thoracic scoliosis and $50^{\circ}$ for lumbar scoliosis.

Another important point in this study was the implementation of a long-term corrective protocol. Many of the previous studies have carried out short-term treatment courses from 6 to 12 weeks $[36,37]$, but due to the progression of curvature in many adolescents, the use of short-term courses will not have much effect. Even if we see a positive effect in the short term, there will be a risk of curve re-progression during the growth period and until the closure of spinal growth plates. Only in a study carried out by Zheng et al. [33], a longer treatment period (12 weeks) was used.

Another factor evaluated in this study was the evaluation of postural control, using SOT, OLST, adaptability, and motion control tests, using a posturography device. Based on the results, there was a significant improvement in the relevant variables in the patient. Following adverse biomechanical changes in the posture, including changes in the center of mass and stretch marks, changes in balance postural and control variables are also expected, which can increase curvature or cause secondary problems such as pain, muscle spasm, postural asymmetry, and muscle imbalance in areas other than the spine $[6,8]$. Therefore, it seems that the examination of postural control during the evaluation and treatment of the spine alignment can reveal useful information about body symmetry and the distribution of the weight in a patient with scoliosis. On the other hand, if a person cannot maintain his/her physical condition in static and dynamic situations due to disturbance in the postural control mechanisms, $\mathrm{s} /$ he cannot benefit from the effects of these therapies [36].

One of the features of corrective exercises in patients with scoliosis is the use of $3 \mathrm{D}$ exercises with the aim of restoring a desired spine alignment, which is usually used to achieve muscle balance. It seems that this restoring affects postural control, and postural control impairment may cause postural deviations or exacerbations of postural abnormalities in these patients. In this regard, in the current study, as well as the Cobb angle measurement, postural control of the patient in different static and dynamic situations was evaluated with the aim of examining the dominant strategies and the sway velocity of the trunk in sagittal and frontal plates.

As expected, in addition to the improvement in Cobb angle, the balance also improved. This may, because of the reduction of spine inclination to kyphosis and scoliosis curves and improvement in trunk symmetry, weight distribution and neuromuscular control following the corrective exercises (dynamic approach) and bracing (static approach). On the other hand, due to the nature of balance exercises performed at the third phase of the program, it seems that the patient's abilities in optimal use of proprioceptive inputs involved in postural con- 
trol have improved. Therefore, the subject is expected to have more control over her physical condition while performing daily tasks, and this may be effective in improving the progression of the patient's postural alignment.

In general, this case study showed that the simultaneous use of bracing and long-term corrective exercises with emphasis on the development of postural control variables was effective in improving the scoliosis curves of an adolescent girl with idiopathic scoliosis. However, it is important to note that despite the positive results in this study, the results of a case study cannot be generalized to all individuals. Although, using these results, it can be argued that in some adolescents with idiopathic scoliosis, it is better to consider other treatment options before surgery. On the other hand, the exercise protocol presented in this case study may be used to treat some adolescents with idiopathic scoliosis that are similar to those of the present study. Hence, it is recommended that, to achieve reliable scientific evidences, more randomized controlled trials should be conducted with the simultaneous use of corrective exercises and bracing in adolescents with idiopathic scoliosis and their long-term outcomes should be examined.

\section{Ethical Considerations}

\section{Compliance with ethical guidelines}

All ethical principles were considered in this article and the participant completed the written consent form.

\section{Funding}

This research did not receive any specific grant from funding agencies in the public, commercial, or non-forprofit sectors.

\section{Authors' contributions}

All authors contributed in designing, running, and writing all parts of the research.

\section{Conflict of interest}

The authors declared no conflict of interest.

\section{References}

[1] Bettany-Saltikov J, Weiss HR, Chockalingam N, Kandasamy G, Arnell T. A comparison of patient-reported outcome measures following different treatment approaches for adolescents with severe idiopathic scoliosis: A system- atic review. Asian Spine Journal. 2016; 10(6):1170-94. [DOI:10.4184/asj.2016.10.6.1170] [PMID] [PMCID]

[2] Gheitasi M, Alizadeh M. H, Rajabi R, Ebrahimi E. [Comparison of the three methods of routine, self-correction and mixed corrective exercise on lateral curvature degree in nonstructural scoliotic subjects (Persian)]. Scientific Journal of Rehabilitation Medicine. 2019; 8(1):47-60.

[3] Gheitasi M, Lordgoee M, Pasandideh Z, Samavi m, Allafan $N$. [Effect of eight-weeks corrective exercises pilates on body dysmorphic metacognitive in female with non-structural scoliosis (Persian)]. Scientific Journal of Rehabilitation Medicine. 2018; 7(4):559-72.

[4] Negrini S, Aulisa AG, Aulisa L, Circo AB, de Mauroy JC, Durmala J, et al. 2011 SOSORT guidelines: Orthopaedic and rehabilitation treatment of idiopathic scoliosis during growth. Scoliosis. 2012; 7:3. [DOI:10.1186/1748-7161-7-3] [PMID] [PMCID]

[5] White AA, Panjabi MM. Clinical biomechanics of the spine. New York: Lippincott; 1990.

[6] Pialasse JP, Mercier P, Descarreaux M, Simoneau M. Sensorimotor control impairment in young adults with idiopathic scoliosis compared with healthy controls. Journal of Manipulative \& Physiological Therapeutics. 2016; 39(7):473-9. [DOI:10.1016/j.jmpt.2016.06.001] [PMID]

[7] Yip J, Liu PY, Yick KL, Cheung MC, Tse CY, Ng SP. Effect of a Functional Garment on Postural Control for Adolescents with Early Scoliosis: A Six-Month Wear Trial Study. In: Advances in Physical Ergonomics and Human Factors. Berlin: Springer; 2016. [DOI:10.1007/978-3-319-41694-6_15]

[8] Pialasse JP, Descarreaux M, Mercier P, Blouin J, Simoneau M. The vestibular-evoked postural response of adolescents with idiopathic scoliosis is altered. PLOS One. 2015; 10(11):e0143124. [DOI:10.1371/journal.pone.0143124] [PMID] [PMCID]

[9] Asher MA, Burton DC. Adolescent idiopathic scoliosis: Natural history and long term treatment effects. Scoliosis. 2006; 1:2. [DOI:10.1186/1748-7161-1-2] [PMID] [PMCID]

[10] Hawes MC, O'Brien JP. The transformation of spinal curvature into spinal deformity: Pathological processes and implications for treatment. Scoliosis. 2006; 1:3. [DOI:10.1186/17487161-1-3] [PMID] [PMCID]

[11] Weiss H-R. Physical therapy intervention studies on idiopathic scoliosis-review with the focus on inclusion criteria 1. Scoliosis. 2012; 7:4. [DOI:10.1186/1748-7161-7-4] [PMID] [PMCID]

[12] Bettany-Saltikov J, Weiss HR, Chockalingam N, Taranu $\mathrm{R}$, Srinivas S, Hogg J, et al. Surgical versus non-surgical interventions in people with adolescent idiopathic scoliosis. Auckland: Cochrane; 2015. [DOI:10.1002/14651858. CD010663.pub2]

[13] Negrini S, Carabalona R. Social acceptability of treatments for adolescent idiopathic scoliosis: A cross-sectional study. Scoliosis. 2006; 1:14. [DOI:10.1186/1748-7161-1-14] [PMID] [PMCID]

[14] Schreiber S, Parent EC, Moez EK, Hedden DM, Hill DL, Moreau M, et al. Schroth physiotherapeutic scoliosis-specific exercises added to the standard of care lead to better cobb angle outcomes in adolescents with idiopathic scoliosis-an 
assessor and statistician blinded randomized controlled trial. PlOS One. 2016; 11(12):e0168746. [DOI:10.1371/journal. pone.0168746] [PMID] [PMCID]

[15] Negrini S, Aulisa L, Ferraro C, Fraschini P, Masiero S, Simonazzi $\mathrm{P}$, et al. Italian guidelines on rehabilitation treatment of adolescents with scoliosis or other spinal deformities. Europa Medicophysica. 2005; 41(2):183-201. [PMID]

[16] Negrini S, Marchini G. Efficacy of the Symmetric, Patientoriented, Rigid, Three-dimensional, active (SPoRT) concept of bracing for scoliosis: A prospective study of the Sforzesco versus Lyon brace. Europa Medicophysica. 2007; 43(2):17181. [PMID]

[17] Coillard C, Leroux MA, Badeaux J, Rivard CH. SPINECOR: A new therapeutic approach for idiopathic scoliosis. Studies in Health Technology and Informatics. 2002; 88:215-7. [PMID]

[18] Smania N, Picelli A, Romano M, Negrini S. Neurophysiological basis of rehabilitation of adolescent idiopathic scoliosis. Disability and Rehabilitation. 2008; 30(10):763-71. [DOI:10.1080/17483100801921311] [PMID]

[19] Negrini S, Minozzi S, Bettany-Saltikov J, Zaina F, Chockalingam N, Grivas TB, et al. Cochrane review: Braces for idiopathic scoliosis in adolescents. Evidence-Based Child Health: A Cochrane Review Journal. 2010; 5(4):1681-720. [DOI:10.1002/ebch.620]

[20] Weiss HR, Negrini S, Hawes MC, Rigo M, Kotwicki T, Grivas TB, et al. Physical exercises in the treatment of idiopathic scoliosis at risk of brace treatment-SOSORT consensus paper 2005. Scoliosis. 2006; 1:6. [DOI:10.1186/1748-7161-1-6] [PMID] [PMCID]

[21] Weiss HR. Rehabilitation of adolescent patients with scoliosis-what do we know? A review of the literature. Pediatric Rehabilitation. 2003; 6(3-4):183-94. [DOI:10.1080/136384903 10001636790] [PMID]

[22] Katz DE, Durrani A. Factors that influence outcome in bracing large curves in patients with adolescent idiopathic scoliosis. Spine. 2001; 26(21):2354-61. [DOI:10.1097/00007632200111010-00012]

[23] Lederman E. Neuromuscular rehabilitation in manual and physical therapies: Principles to practice. London: Churchill Livingstone; 2010. [DOI:10.1016/B978-0-443-06969-7.000140]

[24] de Araújo MEA, da Silva EB, Mello DB, Cader SA, Salgado ASI, Dantas EHM. The effectiveness of the Pilates method: reducing the degree of non-structural scoliosis, and improving flexibility and pain in female college students. Journal of Bodywork and Movement Therapies. 2012; 16(2):191-8. [DOI:10.1016/j.jbmt.2011.04.002] [PMID]

[25] Gheitasi M, Pasandideh M, Lordgoee M, Samavi M, Alafan $\mathrm{N}$. [Effect of eight-weeks pilates exercises on scoliosis deformity and improve balance in female students (Persian)] Journal of Applied Exercise Physiology. 2017; 13(26):61-73.

[26] Gheitasi M, Alizadeh MH, Rajabi R, Ebrahimi E, Haghverdian $\mathrm{S}$. Effect of eight-week routine corrective exercises (stretching-strengthening exercises) on lateral curve of spine in non-structural scoliotic subjects. Journal of Applied Exercise Physiology. 2014; 10(20):93-106.
[27] Schreiber S, Parent EC, Hedden DM, Moreau M, Hill D, Lou E. Effect of schroth exercises on curve characteristics and clinical outcomes in adolescent idiopathic scoliosis: Protocol for a multicentre randomised controlled trial. Journal of Physiotherapy. 2014; 60(4):234. [DOI:10.1016/j. jphys.2014.08.005]

[28] Romano M, Negrini A, Parzini S, Negrini S. Scientific Exercises Approach to Scoliosis (SEAS): Efficacy, efficiency and innovation. Studies in Health Technology and Informatics. 2008; 135:191-207. [PMID]

[29] Kwan KYH, Cheng AC, Koh HY, Chiu AY, Cheung KMC. Effectiveness of Schroth exercises during bracing in adolescent idiopathic scoliosis: Results from a preliminary studySOSORT award 2017 winner. Scoliosis and Spinal Disorders. 2017; 12:32. [DOI:10.1186/s13013-017-0139-6] [PMID] [PMCID]

[30] Weinstein SL, Dolan LA, Wright JG, Dobbs MB. Effects of bracing in adolescents with idiopathic scoliosis. New England Journal of Medicine. 2013; 369(16):1512-21. [DOI:10.1056/NEJMoa1307337] [PMID] [PMCID]

[31] Negrini S, Antonini G, Carabalona R, Minozzi S. Physical exercises as a treatment for adolescent idiopathic scoliosis. A systematic review. Pediatric Rehabilitation. 2003; 6(3-4):22735. [DOI:10.1080/13638490310001636781] [PMID]

[32] Romano M, Minozzi S, Zaina F, Saltikov JB, Chockalingam N, Kotwicki T, et al. Exercises for adolescent idiopathic scoliosis: A Cochrane systematic review. Spine. 2013; 38(14):E883E93. [DOI:10.1097/BRS.0b013e31829459f8] [PMID]

[33] Zheng Y, Dang Y, Yang Y, Li H, Zhang L, Lou EHM, et al. Whether orthotic management and exercise are equally effective to the patients with adolescent idiopathic scoliosis in mainland China?: A randomized controlled trial study. Spine. 2018; 43(9):E494-e503. [DOI:10.1097/ BRS.0000000000002412] [PMID]

[34] Kalichman L, Kendelker L, Bezalel T. Bracing and exercisebased treatment for idiopathic scoliosis. Journal of Bodywork and Movement Therapies. 2016; 20(1):56-64. [DOI:10.1016/j. jbmt.2015.04.007] [PMID]

[35] Negrini S, Donzelli S, Lusini M, Minnella S, Zaina F. The effectiveness of combined bracing and exercise in adolescent idiopathic scoliosis based on SRS and SOSORT criteria: A prospective study. BMC Musculoskeletal Disorders. 2014; 15:263. [DOI:10.1186/1471-2474-15-263] [PMID] [PMCID]

[36] Yagci G, Yakut Y, Simsek E. The effects of exercise on perception of verticality in adolescent idiopathic scoliosis. Physiotherapy Theory and Practice. 2018; 34(8):579-88. [DOI:10.1 080/09593985.2017.1423429] [PMID]

[37] Ko KJ, Kang SJ. Effects of 12-week core stabilization exercise on the cobb angle and lumbar muscle strength of adolescents with idiopathic scoliosis. Journal of Exercise Rehabilitation. 2017; 13(2):244-9. [DOI:10.12965/jer.1734952.476] [PMID] [PMCID] 